\title{
1 Comparison of SARS-CoV-2 Indirect and Direct Detection Methods
}

3 Joel D. Pearson, ${ }^{\mathrm{a}-\mathrm{c}}$ Daniel Trcka, ${ }^{\mathrm{a}}$ Sharon J. Hyduk, ${ }^{\mathrm{d}}$ Marie-Ming Aynaud, ${ }^{\mathrm{a}}$ J. Javier Hernández, 4 Filippos Peidis, ${ }^{\text {a-c }}$ Suying Lu, ${ }^{\mathrm{a}-\mathrm{c}}$ Kin Chan, ${ }^{\mathrm{a}}$ Jim Woodgett, ${ }^{\text {a,f }}$ Tony Mazzulli, ${ }^{\mathrm{c}, \mathrm{g}}$ Liliana Attisano,

7 a. Lunenfeld Tanenbaum Research Institute, Mt Sinai Hospital, Sinai Health System, Toronto,

8 Canada.

9 b. Department of Ophthalmology and Vision Science, University of Toronto, Toronto, Canada

10 c. Department of Laboratory Medicine and Pathobiology, University of Toronto, Toronto, Canada

11 d. Toronto General Hospital Research Institute, University Health Network, Toronto, Canada

12 e. Department of Molecular Genetics, University of Toronto, Toronto, Canada

13 f, Department of Medical Biophysics, University of Toronto, Toronto, Canada

14 g. Department of Microbiology, Sinai Health System/University Health Network, Toronto, Canada

15 h. Department of Biochemistry, Donnelly Centre, University of Toronto, Toronto, Canada 
21 The COVID-19 pandemic caused by the SARS-CoV-2 virus has placed extensive strain on

22 RNA isolation and RT-qPCR reagents. Rapid development of new test kits has helped to

23 alleviate these shortages. However, comparisons of these new detection systems are largely

24 lacking. Here, we compare indirect methods that require RNA extraction, and direct RT-

25 qPCR on patient samples. For RNA isolation we compared four different companies (Qiagen,

26 Invitrogen, BGI and Norgen Biotek). For detection we compared two recently developed

27 Taqman-based modules (BGI and Norgen Biotek), a SYBR green-based approach (NEB

28 Luna Universal One-Step Kit) with published and newly-developed primers, and clinical

29 results (Seegene STARMag RNA extraction system and Allplex 2019-nCoV RT-qPCR assay).

30 Most RNA isolation procedures performed similarly, and while all RT-qPCR modules

31 effectively detected purified viral RNA, the BGI system proved most sensitive, generating

32 comparable results to clinical diagnostic data, and identifying samples ranging from 65 copies

$33-\mathbf{2 . 1 \times 1 0 ^ { 5 }}$ copies of viral Orf1ab/ $\mu$ l. However, the BGI detection system is $\sim \mathbf{4 x}$ more expensive

34 than other options tested here. With direct RT-qPCR we found that simply adding RNase

35 inhibitor greatly improved sensitivity, without need for any other treatments (e.g. lysis buffers

36 or boiling). The best direct methods were $\sim \mathbf{1 0}$ fold less sensitive than indirect methods, but

37 reduce sample handling, as well as assay time and cost. These studies will help guide the

38 selection of COVID-19 detection systems and provide a framework for the comparison of

39 additional systems. 


\section{INTRODUCTION}

41 The SARS-CoV-2 coronavirus is a positive-strand RNA virus with a large genome of about 30kb,

42 which encodes up to 14 open reading frames, including several structural genes; e.g. Nucleocapsid

43 (N), Spike (S), Membrane (M) and Envelope (E), accessory genes, and a large open reading frame

44 (Orf1a/Orf1ab) that encodes a polypeptide that is cleaved into 16 non-structural proteins $(1,2)$. It is

45 related to the SARS-CoV and MERS-CoV coronaviruses, which cause severe respiratory illness in

46 humans, and is the causative agent of the COVID-19 respiratory disease (3). Since the first

47 documented case in Wuhan, China in December 2019, the virus has spread rapidly across the globe.

48 On March 11, 2020, the WHO officially declared COVID-19 a pandemic (4, 5). As of May 12,

49 2020, there have been over 4.2 million reported cases of COVID-19 and over 286,000 deaths

50 worldwide (6).

51 The wide range of disease symptoms, including a large portion of mildly or asymptomatic people,

52 has facilitated rapid dissemination $(7,8)$. Efficient diagnosis, allowing rapid and accurate patient

53 testing remains the key to limiting disease spread. Rapid disease spread has strained the capacity of

54 diagnostic facilities, and the availability of standard reagents. The principle means of diagnostics for

55 COVID-19 relies on RNA extraction from nasal swabs followed by reverse transcriptase-

56 quantitative PCR (RT-qPCR) detection of viral genes (e.g. N, E and RdRp). Rapid development of

57 SARS-CoV-2 RT-qPCR detection systems from many companies has helped to alleviate some of

58 the strain, and many new systems have been given Emergency Use Authorization (EUA) for clinical

59 use. However, comparison of new systems with clinical diagnostics is largely lacking. A limited

60 number of studies have evaluated some kits and compared efficiency of different RT-qPCR primer

61 sets for COVID-19 detection (9-12). These studies have revealed large differences in sensitivity of 
62 some systems, highlighting the need for stringent comparison and further optimization of novel

63 detection systems.

64 An attractive option is direct detection from patient samples without RNA extraction, as it increases

65 throughput, decreases costs and circumvents the need for clinically approved RNA extraction

66 reagents which have become limited. Several studies have examined the ability to directly detect

67 patient samples collected in UTM. While Grant et al. reported no effect on sensitivity with

68 extraction-free COVID-19 detection (13), several other studies noted a decrease in the order of 5-20

69 fold in sensitivity (14-18). Interestingly, while Grant et al. observed reduced detection sensitivity

70 after heating the sample to $95^{\circ} \mathrm{C}(13)$, others have demonstrated that heating samples to $95^{\circ} \mathrm{C}$ could

71 partially increase sensitivity (14-16), as could detergent-based lysis $(16,19)$. In studies where large

72 sample numbers were analyzed, optimized extraction-free methods resulted in a high (92-98\%)

73 concordance with clinical results, despite reduced sensitivity (14-16).

74 Here, we comprehensively compare two recently developed COVID-19 detection protocols, one

75 from BGI and the other from Norgen Biotek. The BGI system has been used extensively in China,

76 and has recently been approved for use in several other countries, whereas the Norgen System is

77 currently seeking approval for use starting in Canada. We compare the RNA isolation systems from

78 both companies alongside the Qiagen RNeasy and Invitrogen Purelink systems, both of which are

79 routinely used in research labs, and the former of which has been shown to provide only modestly

80 reduced recovery compared to the CDC approved QIAamp Viral RNA kit (17). We also compare

81 and optimize BGI and Norgen Taqman RT-qPCR detection modules, as well as a SYBR green-

82 based protocol using a commercially available RT-qPCR mix with published and newly designed

83 primer sets. In addition, we evaluate and optimize the ability of the BGI and Norgen systems to

84 detect COVID-19 directly from patient swabs collected in UTM, without RNA extraction. Finally, 
85 we perform a cost analysis and discuss both advantages and drawbacks of both systems. We find

86 that both Norgen and BGI RNA isolation kits perform similarly to the Qiagen RNeasy system,

87 while the Invitrogen Purelink is less efficient. We also find that the significantly more expensive,

88 and less flexible BGI RT-qPCR detection module is the most sensitive of the systems tested,

89 providing comparable results to clinical diagnostic data, and could efficiently diagnose patients

90 using extraction-free detection. While initially less sensitive, we found that sensitivity of the more

91 affordable, and flexible Norgen RT-qPCR module could be dramatically improved to levels

92 matching the BGI mix in direct detection assays simply by adding an RNase inhibitor. 


\section{MATERIALS AND METHODS}

94 Patient samples. Samples were obtained from the MSH/UHN clinical diagnostics lab with

95 approvals from the Research Ethics Boards (REB \#20-0078-E) of Mount Sinai Hospital in Toronto,

96 Canada. Clinical diagnostic data was obtained using the Seegene STARMag RNA extraction kit

97 (Microlab STAR Liquid Handling System, Hamilton Company) and Allplex 2019-nCoV RT-qPCR

98 assay analyzed using the Bio-Rad CFX96 IVD real-time qPCR detection system.

99 RNA extraction. Qiagen RNeasy, Invitrogen Purelink, Norgen Biotek Total RNA Purification Kit

100 and the BGI Magnetic Bead Viral RNA/DNA extraction kit were used as per manufacturer's

101 protocols. For each extraction, $100 \mu \mathrm{l}$ of sample was used and eluted in $32 \mu 1$.

Taqman-based RT-qPCR detection. The 2019-nCoV TaqMan RT-PCR Kit from Norgen Biotek and 2019-nCoV: Real-Time Fluorescent RT-PCR kit from BGI were used essentially as per manufacturer's instructions. For comparison of different plate formats (Fig. 1A), 10 and $20 \mu 1$ reaction volumes were used with either 2.5 or $5 \mu 1$ synthetic RNA standard (Twist Biosci.), respectively, using the Norgen system. These were analyzed in parallel on separate BioRad CFX96

107 (20 $\mu \mathrm{l}$ reactions) or CFX384 (10 $\mu$ l reactions) real-time PCR systems. All other experiments used 10 $108 \mu \mathrm{l}$ reaction volumes (384-well plates) with $2.5 \mu \mathrm{l}$ of sample (synthetic standard, extracted RNA or direct UTM) and were analyzed using a Bio-Rad CFX384 detection system. For testing alternative

110 primers/probes with the Norgen system, primers/probes were purchased from Integrated DNA

111 Technologies (IDT) and primers were used at $500 \mathrm{nM}$ with probes at $250 \mathrm{nM}$. Probes were FAM-

112 labelled, E Sarbeco and HKU Orf1 sequences are published $(9,12)$, and newly designed N gene

113 primers/probe (N Pearson) sequences are Fwd: CCAGAATGGAGAACGCAGT, Rev:

114 TGAGAGCGGTGAACCAAGA, probe: GCGATCAAAACAACGTCGGCCCC). RT-qPCR 
115 cycling protocols were as per manufacturers recommendations, except for annealing/elongation

116 temperature testing (Fig. 2E) with the Norgen system where the indicated temperatures were used.

117 SYBR green RT-qPCR detection. Primer pairs were designed using PrimerQuest software, and

118 purchased from IDT. Primers selected for testing had $\Delta \mathrm{G}$ values for self-dimers and heterodimers

119 greater than $-9.0 \mathrm{kcal} /$ mole. Newly designed primers were specific for SARS-CoV-2 with no cross-

120 reactivity to other coronaviruses based on published sequences (SH N1 Fwd:

121 AATTGCACAATTTGCCCCCA, Rev: ACCTGTGTAGGTCAACCACG; SH S1 Fwd:

122 TCAGACAAATCGCTCCAGGG, Rev: TCCAAGCTATAACGCAGCCT). The published S gene

123 primers used in this study were S1 Fwd: CCTACTAAATTAAATGATCTCTGCTTTACT, Rev:

124 CAAGCTATAACGCAGCCTGTA (20). Primers were used at $400 \mathrm{nM}$. RT-qPCR was performed

125 on a LightCycler 480 (Roche) with a 384 well plate using the NEB Luna Universal One-Step RT-

126 qPCR kit (NEB \#E3005L, New England Biolabs Inc) and a reaction volume of $10 \mu 1$ with $2.5 \mu 1$ of

127 sample. Cycling conditions were as follows: 10 min @ $55^{\circ} \mathrm{C}(\mathrm{RT}), 1$ min @ $95^{\circ} \mathrm{C}$ (denaturation), 45

128 cycles: 10 s @ 95 $\mathrm{C}, 30$ s @ 60 $\mathrm{C}$ (amplification), melt curve. Standard curves were generated for

129 each primer set with serial dilutions of viral RNA from 0.8 to 800,000 copies/ $\mu$ l; SARS-CoV-2

130 RNA (strain USA_WA1/2020) was provided by the World Reference Centre for Emerging Viruses

131 and Arboviruses (Galveston, TX) (WRCEVA).

132 Direct extraction-free SARS-CoV-2 detection. For direct detection, $2.5 \mu$ l of patient sample in

133 universal transport media (UTM, Copan) were added to the RT-qPCR reaction mix. For comparison

134 to extracted RNA, an equivalent input of extracted RNA was used (i.e. extracted RNA eluted in 32

$135 \mu$ l was diluted 1:2 with RNase-free water). To optimize direct detection, RNaseOUT

136 (ThermoFisher) was added to UTM samples (2 U/ $\mu 1)$. Samples were then left untreated, heated at

$13795^{\circ} \mathrm{C}$ for 15 min, mixed 1:1 with Lucigen QuickExtract DNA extraction solution with heating at 
bioRxiv preprint doi: https://doi.org/10.1101/2020.05.12.092387; this version posted May 13, 2020. The copyright holder for this preprint (which

was not certified by peer review) is the author/funder, who has granted bioRxiv a license to display the preprint in perpetuity. It is made available under aCC-BY-ND 4.0 International license.

$13895^{\circ} \mathrm{C}$ for $5 \mathrm{~min}$ or treated with MyPOLS Bio VolcanoCell2G lysis buffer, $1 \%$ Triton X-100, $1 \%$

139 Tween-20 or $1 \%$ Saponin and incubated on ice $15 \mathrm{~min}$. Samples were then directed added to the RT-

140 qPCR reaction mixture and compared to UTM samples that had been left untreated. 


\section{RESULTS}

142 Plate format. Many diagnostic protocols utilize $20 \mu 1$ reactions in 96-well plates, but reducing

143 volume in a 384-well format increases throughput and reduces costs. Using the Norgen RT-qPCR

144 COVID-19 detection kit (which utilizes CDC-approved N1 and N2 primers), we observed similar

$145 \mathrm{Ct}$ values in a comparison of 20 vs $10 \mu \mathrm{l}$ reactions in 96- or 384-well plates, respectively (Fig. 1A),

146 thus in subsequent analysis we focused on 384-well plates.

147 RNA Extraction Methods. Qiagen RNA extraction systems are used extensively for viral RNA

148 isolation, but availability has become limited. Thus, we first compared the Qiagen RNeasy RNA

149 extraction kit to a similar kit from Norgen Biotek, both of which utilize silica-based columns. None

150 of the SARS-CoV-2-negative samples generated any signal, and we detected no significant

151 difference in Ct values across four clinically-diagnosed positive patient samples (Fig. 1B), thus the

152 Norgen extraction system performs similarly to standard Qiagen kits. We next compared efficiency

153 of the Norgen (column based), Invitrogen Purelink (column-based) and BGI (magnetic bead-based)

154 RNA isolation systems. Using two new positive patient samples, we observed similar recovery with

155 both the Norgen and BGI systems, but considerably higher Ct values were observed for viral (N1

156 and N2 primers) and human control (RNase P) genes with the Invitrogen kit (Fig. 1C). Thus, for

157 isolating SARS-CoV-2 RNA from nasopharyngeal patient samples in UTM, Norgen, Qiagen

158 RNeasy and BGI extraction methods are all comparable, but the Invitrogen Purelink kit is less

159 efficient.

160 TaqMan Primers/RT-qPCR mix. Next we compared the efficiency of two recently developed

161 TaqMan-based SARS-CoV-2 RT-qPCR detection kits from Norgen and BGI. The BGI protocol

162 uses one primer set against Orf1ab, while as noted above, Norgen uses two separate reactions 
163 targeting the $\mathrm{N}$ gene. We first compared RNA from the two positive patient samples extracted with

164 the Norgen, BGI or Invitrogen Purelink methods, as well as RNA isolated from one negative patient

165 sample using the BGI extraction method. These assays confirmed that Norgen/BGI extraction

166 methods are more efficient than Purelink, but also revealed greater sensitivity $(\sim 1-3 \mathrm{Ct}$ values $)$ with

167 BGI vs Norgen primers/RT-qPCR mix, particularly with the lowest level sample (L028-Purelink)

168 (Fig. 2A). We observed a similar trend across seven additional samples all isolated using the

169 Norgen RNA extraction kit (Fig. 2B). Comparison to clinical values for the viral N, E and RdRp

170 genes obtained using the Seegene STARMag RNA extraction kit and Allplex 2019-nCoV RT-qPCR

171 assay analyzed using the Bio-Rad CFX96 IVD real-time qPCR detection system, showed a strong

172 correlation with $\mathrm{Ct}$ values obtained using either the BGI or Norgen detection modules (Fig. 2C). We

173 observed no significant difference between the BGI and clinical values, although there was a trend

174 toward lower $\mathrm{Ct}$ values with the clinical lab E gene primers/probe, particularly with higher-level

175 samples (Fig. 2C). In contrast, the Norgen detection module showed significantly higher Ct values

176 compared to clinical data (median 1.4 to $3.5 \mathrm{Ct}$ values higher depending on the primer sets used)

177 (Fig. 2C), similar to what we observed in comparison to the BGI detection system. Using synthetic

178 TWIST Bioscience SARS-CoV-2 standards we found that the BGI detection kit routinely detected

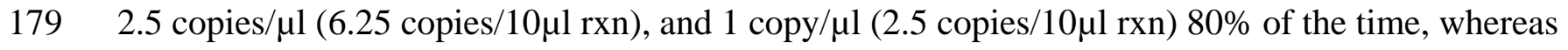

180 the detection limit of the Norgen system was 10 copies/ $\mu 1$ (25 copies/10 $\mu 1$ rxn) (Fig. 2D), with

181 lower concentrations being detected $<50 \%$ of the time.

182 To determine if sensitivity using the Norgen RT-qPCR mix could be enhanced, we tested different

183 annealing/elongation temperatures in the qPCR reaction along with two other published SARS-

184 CoV-2 primers/probes shown to have high sensitivity (E Sarbeco and HKU Orf1) $(9,12,21,22)$,

185 and new primers/probes we designed to target the viral $\mathrm{N}$ gene. Increasing the annealing/elongation 
temperature from the manufacturer's recommended $55^{\circ} \mathrm{C}$ did not affect $\mathrm{Ct}$ values for either the $\mathrm{N} 1$

187 or N2 primers provided with the Norgen system (Fig. 2E). Using the Norgen RT-qPCR mix, we

188 observed poor performance of the HKU Orf1 primer set, and the newly designed $\mathrm{N}$ gene primers

189 provided higher $\mathrm{Ct}$ values compared to the CDC N1 and N2 primers, but the E gene primers/probes

190 demonstrated lower (more sensitive) $\mathrm{Ct}$ values compared to the $\mathrm{N} 1 / \mathrm{N} 2$ primers, particularly at $59^{\circ} \mathrm{C}$

191 annealing/elongation (Fig. 2E). This improvement, however, did not translate to a lower detection

192 limit (Fig. 2D). Thus, while both systems easily detect purified SARS-CoV-2 RNA from infected

193 patients, the BGI primers/RT-qPCR system provides a lower detection limit and similar Ct values to

194 clinical data, while Ct values for the Norgen detection module are $\sim 2-3$ cycles higher.

195 SYBR green detection. We next compared the more sensitive BGI detection system to a SYBR

196 green-based method. We tested various published primers, some designed for SYBR green and

197 some from TaqMan assays $(9,12,20,23)$, and designed our own. One published set for the viral S

198 gene (20) and two new N or S gene primer sets gave little/no signal in no-template control (NTC)

199 and generated a linear response across 8 - 800,000 viral copies/ $\mu$ (Fig. S1A), and were thus selected

200 for future analyses. We then compared SARS-CoV-2 standards using the SYBR green primers and

201 the BGI detection kit and observed comparable Ct values between the two systems across 20 to

202 20,000 genome copies/ $\mu$ l (Fig. 3A). Identical Ct values were obtained using SARS-CoV-2 RNA

203 from WRCEVA (not shown). The BGI system provided a slightly lower detection limit than the

204 SYBR green systems (compare Figs. 2D and 3D).

205 We next analyzed patient samples comparing the SYBR green primers to previous data obtained

206 with the BGI kit (Fig. 3C). One of the primers (SH S1) did not perform well on patient samples and

207 was excluded from these experiments. The other SYBR green primers reliably identified all 
negative and positive patient samples, with $\mathrm{SH}-\mathrm{N} 1$ primers generating slightly lower $\mathrm{Ct}$ values $(0.3$ to $1.1 \mathrm{Ct}$ values, $\mathrm{p}=0.02$ ) and $\mathrm{S} 1$ primers providing slightly higher $\mathrm{Ct}$ values compared to the BGI system $(-0.2$ to $1.6 \mathrm{Ct}$ values, $\mathrm{p}<0.01)$. Quantification of gene copy numbers generated similar

211 results for SYBR green and BGI, and ranged from 24 copies to >120,000 copies/ $\mu 1$ (Fig. 3D). Non-

212 specific melt peaks were occasionally observed in negative and low virus copy positive samples,

213 which could easily be identified and excluded (Fig. S1B). All patient samples were positive for 214 human RNase P (not shown).

215 One step detection without RNA purification. To reduce the number of steps required for viral 216 detection we tested RT-qPCR direct from patient samples in UTM. For this, we added $2.5 \mu$ of 217 sample directly to the RT-qPCR mix and compared this to an equivalent input of extracted RNA.

218 UTM blocked SYBR-green detection of SARS-CoV-2 RNA standards (data not shown), but both 219 the BGI and Norgen TaqMan detection systems identified positive patient samples (Fig. 4A). Ct 220 values were lower for BGI vs Norgen, consistent with data with purified RNA (c.f. Figs. 2 \& 4A).

221 Furthermore, the Norgen system did not reliably identify some positive samples with lower levels of

222 virus (Fig. 4A). Relative to extracted RNA, direct RT-qPCR with the BGI detection kit was 2-26

223 fold less sensitive (except sample L021, which was $\sim 600$-fold reduced, see below for an

224 explanation), whereas with the Norgen kit it was 20-1000's fold lower (L033 with the N2 primers 225 was an exception at 4.4-fold). Despite the reduced sensitivity, the strong correlation between BGI 226 and clinical Ct values was maintained (Fig. 4B).

227 Others have reported that reduced sensitivity in direct $v s$. extracted RNA analyses can be partially 228 overcome by heat or different lysis buffers/detergents $(14-16,19)$. Thus, we assessed the effect of 229 adding an RNase inhibitor (RNaseOUT), heating samples at $95^{\circ} \mathrm{C}$ for 15 minutes, or five different 230 lysis buffers/detergents (Lucigen QuickExtract DNA extraction solution, MyPOLS Bio 
231 VolcanoCell2G lysis buffer, 1\% Triton X-100, 1\% Tween-20 or 1\% Saponin). Simply adding

232 RNase inhibitor was sufficient to dramatically increase detection $>100$ fold using the Norgen

233 system, bringing Ct values to levels comparable to those obtained with the BGI RT-qPCR system,

234 and, most importantly, allowing for detection of previous "false-negative" samples L021 and L032

235 (Fig. 4C). Furthermore, RNase inhibitor brought direct RT-qPCR results with the Norgen detection

236 kit to within $3 \mathrm{Ct}$ values ( 10-fold) of those obtained with extracted RNA (compare Figs. 4A and

237 C). Treatment with heat, lysis buffers or detergents did not appreciably increase sensitivity further,

238 and in some cases reduced sensitivity (higher Ct values). For the BGI detection system, none of the

239 treatments dramatically improved detection, with the exception of sample L021 (Fig. 4C), which

240 previously showed the largest difference between extracted RNA and direct UTM analysis (Fig.

$2414 \mathrm{~A})$. We presume, therefore, that L021 had higher RNase levels that were not fully inhibited by the

242 (proprietary) RNase inhibitor already present in the BGI mix. Thus, RNase inhibitor is sufficient to

243 improve direct detection and under these conditions BGI and Norgen kits perform similarly.

\section{DISCUSSION}

245 Here, we comprehensively compared four different RNA isolation methods, two recently released

246 SARS-CoV-2 TaqMan RT-qPCR detection modules and a SYBR green-based RT-qPCR approach

247 for SARS-CoV-2 detection using published and newly-developed primers. Furthermore, we tested

248 and optimized extraction-free SARS-CoV-2 detection using these same detection modules.

249 For RNA extraction, we tested three different column-based systems from Qiagen (RNeasy),

250 Invitrogen (Purelink) and Norgen Biotek, as well as a magnetic silica bead system from BGI. While

251 only the BGI system is specifically marketed for viral RNA isolation, we observed similar results

252 using the Qiagen RNeasy, Norgen and BGI systems, and while it was only tested on two samples, 
we observed lower recovery of viral RNA using the Invitrogen Purelink system. Cost analysis of the

254 BGI and Norgen Biotek RNA isolation systems revealed that the latter is $\sim 40 \%$ more expensive

255 than that of BGI (\$6.55 CAD vs. \$4.68 CAD/sample, Fig. 4D), but we found that for small batches

256 of samples the bead-based BGI kit was slower, increasing sample preparation time by about $50 \%$

257 over the Norgen kit ( 30 vs. $45 \mathrm{~min})$. This difference was largely due to two incubation steps in the

258 BGI protocol, so the relative difference in sample preparation time may diminish as larger numbers

259 of samples are processed. Furthermore, magnetic beads facilitates large-scale, automated sample

260 extraction.

261 For RNA detection, we tested TaqMan-based detection systems from BGI and Norgen Biotek, as

262 well as a SYBR green method using a commercially available RT-qPCR mix and published primers

263 (some used for SYBR green and others from probe-based methods) along with new primers we

264 developed. All systems could accurately detect SARS-CoV-2 positive patient samples using

265 extracted RNA, and generated Ct values that strongly correlated with clinical diagnostic values.

266 However, the BGI and SYBR green methods routinely produced lower Ct values for patient

267 samples, which closely match clinical results, and had lower detection limits compared to the

268 Norgen system. The BGI system also performed slightly better that the SYBR green methods with

269 low-level standards. One drawback to the SYBR green method was reduced specificity, as we

270 sometimes observed non-specific products in negative or low-level samples, although these could

271 be identified by monitoring melt curves. These non-specific products were not routinely observed in

272 NTC reactions. Thus, melt curve analysis is an essential component of SYBR green qPCR. We also

273 tested 8 other published and newly designed primers and all yielded non-specific PCR products (not

274 shown). Whether non-specific products can be eliminated using alternative RT-qPCR mixes

275 remains to be determined. The BGI detection module is over four-times more expensive than 
276 Norgen or SYBR green methods (Fig. 4D), providing a significant financial drawback. Cost savings

277 with the Norgen kit could be even greater if multiplexing primers/probes were utilized; currently

278 this system follows the CDC guidelines with three separate reactions, one each using FAM-labelled

279 viral N1, viral N2 or human RNase P primers/probes. The Norgen and SYBR green systems also

280 provide more flexibility than that of BGI. Primers/probes come pre-mixed in the BGI system and

281 cannot be altered, whereas they are added separately in the others, allowing alternative primer/probe

282 options and concentrations. We tested three alternative primers/probes with the Norgen system.

283 Those targeting the E gene performed similarly to the provided N1/N2 primers/probes, while

284 alternatives for the viral $\mathrm{N}$ or Orfla gene had reduced sensitivity, although only a single

285 primer/probe concentration was tested. Sequences of the BGI primers/probes are unavailable, and

286 only a single primer/probe set targeting the viral Orflab gene is used. Mutation could affect

287 detection and generate false negatives. Thus, while the BGI system provides a lower detection limit

288 with extracted RNA than Norgen or SYBR green detection systems, all accurately identified SARS-

289 CoV-2-positive patients, and latter systems detect multiple viral targets and offer greater flexibility

290 and substantially reduced costs.

291 Finally, we tested direct, extraction-free detection of SARS-CoV-2. This approach reduces cost,

292 increases throughput, and circumvents the need for RNA extraction systems that may be scarce

293 during a pandemic. Others have shown that SARS-CoV-2 can be detected from patient samples,

294 although this typically comes with reduced sensitivity, which can at least partially be overcome by

295 heat and/or detergent lysis (14-16, 19). We found that SYBR green-based detection was

296 incompatible with direct detection of samples in UTM. The unmodified BGI detection system

297 performed well in the direct detection of unprocessed patient samples, and confirmed all positive

298 samples tested across a wide range of clinical values, but had a reduced median sensitivity of $\sim 12-$ 
299 fold compared to extracted RNA. The Norgen system initially performed poorly on direct UTM

300 samples, generating much higher Ct values than extracted RNA (in some cases 1000s of fold

301 higher), and resulted in several false-negatives. Critically, however, adding RNase inhibitor

302 increased sensitivity of direct RT-qPCR with the Norgen system > 100-fold, allowing detection of

303 all previously false-negative samples. This modification did not, in most cases, dramatically

304 increase sensitivity of direct sample analysis with the BGI detection system, suggesting it already

305 contains an RNase inhibitor. Even in that case however, detection of one patient sample was

306 markedly improved, implying higher RNase levels. Thus, addition of RNase inhibitor is a simple

307 and sufficient step to facilitate diagnosis of SARS-CoV-2 direct from patient samples.

308 Our results provide in depth analysis of recently released SARS-CoV-2 detection systems from BGI

309 and Norgen Biotek and compare these to a SYBR green-based approach and to clinical diagnostic

310 values. Each system provides advantages and disadvantages depending on sensitivity, specificity,

311 flexibility and cost. Our findings will help guide selection of SARS-CoV-2 detection systems, and

312 provide an outline for others to compare alternative systems.

\section{ACKNOWLEDGEMENTS}

314 This work was funded by a grant to RB, JW and LP from the Krembil Foundation,

315 and the Canadian Institutes of Health Research (CIHR; FDN-154299) to MIC. 


\section{REFERENCES}

317 1. Wu F, Zhao S, Yu B, Chen Y-M, Wang W, Song Z-G, Hu Y, Tao Z-W, Tian J-H, Pei Y-Y,

318 Yuan M-L, Zhang Y-L, Dai F-H, Liu Y, Wang Q-M, Zheng J-J, Xu L, Holmes EC, Zhang Y-

319 Z. 2020. A new coronavirus associated with human respiratory disease in China. Nature

$320 \quad 579: 265-269$.

2. Chan JF-W, Kok K-H, Zhu Z, Chu H, To KK-W, Yuan S, Yuen K-Y. 2020. Genomic characterization of the 2019 novel human-pathogenic coronavirus isolated from a patient with atypical pneumonia after visiting Wuhan. Emerg Microbes Infect 9:221-236.

3. Wu A, Peng Y, Huang B, Ding X, Wang X, Niu P, Meng J, Zhu Z, Zhang Z, Wang J, Sheng J, Quan L, Xia Z, Tan W, Cheng G, Jiang T. 2020. Genome Composition and Divergence of the Novel Coronavirus (2019-nCoV) Originating in China. Cell Host Microbe 27:325-328.

4. Coronavirus disease 2019.

5. WHO Director-General's opening remarks at the media briefing on COVID-19 - 11 March 2020.

6. COVID-19 Map - Johns Hopkins Coronavirus Resource Center.

331 7. Day M. 2020. Covid-19: identifying and isolating asymptomatic people helped eliminate virus 332 in Italian village. BMJ 368:m1165.

333 8. Lauer SA, Grantz KH, Bi Q, Jones FK, Zheng Q, Meredith HR, Azman AS, Reich NG, 334 Lessler J. 2020. The Incubation Period of Coronavirus Disease 2019 (COVID-19) From 335 Publicly Reported Confirmed Cases: Estimation and Application. Ann Intern Med. 
9. Nalla AK, Casto AM, Huang M-LW, Perchetti GA, Sampoleo R, Shrestha L, Wei Y, Zhu H, Jerome KR, Greninger AL. 2020. Comparative Performance of SARS-CoV-2 Detection Assays using Seven Different Primer/Probe Sets and One Assay Kit. J Clin Microbiol.

10. Wang X, Yao H, Xu X, Zhang P, Zhang M, Shao J, Xiao Y, Wang H. 2020. Limits of Detection of Six Approved RT-PCR Kits for the Novel SARS-coronavirus-2 (SARS-CoV-2). Clin Chem.

11. Zhen W, Manji R, Smith E, Berry GJ. 2020. Comparison of Four Molecular In Vitro Diagnostic Assays for the Detection of SARS-CoV-2 in Nasopharyngeal Specimens. J Clin Microbiol.

12. Vogels CBF, Brito AF, Wyllie AL, Fauver JR, Ott IM, Kalinich CC, Petrone ME, CasanovasMassana A, Muenker MC, Moore AJ, Klein J, Lu P, Lu-Culligan A, Jiang X, Kim DJ, Kudo E, Mao T, Moriyama M, Oh JE, Park A, Silva J, Song E, Takehashi T, Taura M, Tokuyama M, Venkataraman A, Weizman O-E, Wong P, Yang Y, Cheemarla NR, White E, Lapidus S, Earnest R, Geng B, Vijayakumar P, Odio C, Fournier J, Bermejo S, Farhadian S, Cruz CD, (SARS-CoV-2) diagnosis by RT-PCR to increase capacity for national testing programmes during a pandemic. bioRxiv 2020.04.06.028316. 
14. Fomsgaard AS, Rosenstierne MW. 2020. An alternative workflow for molecular detection of SARS-CoV-2 - escape from the NA extraction kit-shortage, Copenhagen, Denmark, March 2020. Euro Surveill 25.

15. Bruce EA, Huang M-L, Perchetti GA, Tighe S, Laaguiby P, Hoffman JJ, Gerrard DL, Nalla AK, Wei Y, Greninger AL, Diehl SA, Shirley DJ, Leonard DGB, Huston CD, Kirkpatrick BD, Dragon JA, Crothers JW, Jerome KR, Botten JW. 2020. DIRECT RT-qPCR DETECTION OF

16. Massive and rapid COVID-19 testing is feasible by extraction-free SARS-CoV-2 RT-qPCR | SARS-CoV-2 RNA FROM PATIENT NASOPHARYNGEAL SWABS WITHOUT AN RNA

17. RT-qPCR DETECTION OF SARS-CoV-2 RNA FROM PATIENT NASOPHARYNGEAL SWAB USING QIAGEN RNEASY KITS OR DIRECTLY VIA OMISSION OF AN RNA EXTRACTION STEP. bioRxiv 2020.03.20.001008.

18. Beltrán-Pavez C, Márquez CL, Muñoz G, Valiente-Echeverría F, Gaggero A, Soto-Rifo R, Barriga GP. 2020. SARS-CoV-2 detection from nasopharyngeal swab samples without RNA extraction. bioRxiv 2020.03.28.013508.

19. Ladha A, Joung J, Abudayyeh O, Gootenberg J, Zhang F. 2020. A 5-min RNA preparation method for COVID-19 detection with RT-qPCR. medRxiv 2020.05.07.20055947. 
2019 novel coronavirus indicating person-to-person transmission: a study of a family cluster.

379 21. Corman VM, Landt O, Kaiser M, Molenkamp R, Meijer A, Chu DK, Bleicker T, Brünink S,

380 Schneider J, Schmidt ML, Mulders DG, Haagmans BL, van der Veer B, van den Brink S,

381 Wijsman L, Goderski G, Romette J-L, Ellis J, Zambon M, Peiris M, Goossens H, Reusken C,

382 Koopmans MP, Drosten C. 2020. Detection of 2019 novel coronavirus (2019-nCoV) by real-

383 time RT-PCR. Euro Surveill 25.

384 22. Chu DKW, Pan Y, Cheng SMS, Hui KPY, Krishnan P, Liu Y, Ng DYM, Wan CKC, Yang P,

385 Wang Q, Peiris M, Poon LLM. 2020. Molecular Diagnosis of a Novel Coronavirus (2019-

386 nCoV) Causing an Outbreak of Pneumonia. Clin Chem.

23. Won J, Lee S, Park M, Kim TY, Park MG, Choi BY, Kim D, Chang H, Kim VN, Lee CJ. 


\section{FIGURE LEGENDS}

\section{FIG 1: Comparison of plate formats and RNA extraction kits.}

393 (A) Serial dilutions of SARS-CoV-2 synthetic RNA standards from Twist Biosci (in copies/ $\mu$ of the

394 standard added to the RT-qPCR reaction) run in parallel on separate BioRad CFX 96-well (20 $\mu 1$

395 reactions) or 384-well (10 $\mu$ l reactions) real-time PCR systems using the Norgen COVID-19 RT-

396 qPCR detection module. Mean +/- range of two independent tests. (B-C) Analysis of four negative

397 and four positive patient samples extracted with either the Qiagen RNeasy or Norgen RNA isolation

398 kits (B) or two positive patient samples extracted with the Norgen, Invitrogen Purelink or BGI RNA

399 isolation kits (C) using the Norgen RT-qPCR detection system and N1, N2 or human control (Rnase

400 P) primers. Samples L015, L018 and L019 are the mean +/- range of technical duplicates run

401 independently on two separate plates, other samples were analyzed once, although L028 and L029

402 are rerun in Fig. 2a. In (B) a paired t-test was used to compare Norgen $v s$. Qiagen extractions.

404 FIG 2: BGI detection kit shows enhanced sensitivity over Norgen kit.

405 (A) Analysis of two positive patient samples extracted with the BGI, Norgen (Nor) or Invitrogen

406 Purelink (Pure) RNA isolation kits using the Norgen (N1 or N2 primers) or BGI RT-qPCR

407 detection systems. Mean $+/$ - std dev of the same sample run on three (BGI \& Norgen extractions) or

408 two (Purelink extraction) separate plates. (B) Analysis of additional patient samples using the

409 Norgen (N1 or N2 primers) or BGI detection systems. Mean +/- range of the same samples run

410 independently on two separate plates. Paired t-tests compare Norgen N1 or N2 and BGI Ct values

411 across all samples (including L028 and L029 extracted with the Norgen kit). (C) Comparison of Ct

412 values from clinical lab analysis (E, RdRp and N genes) and data obtained with the BGI or Norgen

413 detection systems. Paired t-tests were used to compare results. (D) Detection limit determination 
414 using the BGI or Norgen detection systems shown as the number of positives/total number of wells.

415 Concentrations are in copies/ $\mu$ in the standard. N/D: not determined. (E) Analysis of 500 viral

416 copies (Twist Biosci) using N1, N2, E Sarbeco, HKU Orf1 and our N gene (N_Pearson) and the

417 Norgen RT-qPCR mix with the indicated annealing/elongation temperatures. Mean +/- range of two

418 independent tests.

FIG 3. SYBR green detection of extracted RNA.

421 (A) Serial dilutions of SARS-CoV-2 synthetic RNA standards (Twist Biosci) were run in SYBR

422 green and BGI TaqMan assays. Mean + /- STD; $n \geq 3$. (B) Detection limit for each of the SYBR

423 green primer sets shown as the number of positive samples/total number of samples tested.

424 Synthetic RNA (Twist Biosci) was used from stocks with the indicated number of copies per $\mu \mathrm{L}$.

425 (C) Comparison of Ct values obtained for each patient sample with the SYBR green and BGI

426 TaqMan assays. Linear regression was used to determine the $\mathrm{R}^{2}$. BGI data is from Fig. 2A, B. (D)

427 Comparison of viral copy number per $\mu \mathrm{L}$ for each of the positive patient samples determined with

428 each primer set. Copy number was determined using a standard curve of SARS-CoV-2 RNA

429 (WRCEVA).

$431 \quad$ FIG 4: One-step direct detection without RNA extraction.

432 (A) Analysis of extracted RNA or direct UTM from a panel of patients using the BGI or Norgen

433 (N1 and N2 primers) detection systems. (B) Comparison of Ct values from clinical lab analysis on

434 extracted RNA (E, RdRp and N genes) to data obtained for direct analysis with the BGI detection

435 system. (C) Patient samples in UTM were left untreated, or treated with the RNase inhibitor

436 RNaseOUT with or without heating at $95^{\circ} \mathrm{C}$ for $15 \mathrm{~min}$, or treated with the indicated lysis 
437 buffers/detergents and then directly analyzed using the BGI or Norgen (N1/N2 primers) RT-qPCR

438 detection systems. Note sample L020 (clinical negative) was also tested under these conditions and

439 was confirmed as SARS-CoV-2 negative. (D) Cost analysis comparing Norgen, BGI, and SYBR

440 green systems. Price is in CAD at the time these studies were initiated (late March/early April 2020)

441 for $10 \mu 1 \mathrm{RT}-\mathrm{qPCR}$ reactions and include relevant processing and shipping fees. * BGI RNA

442 extraction module is based on the 96-sample format, price can be reduced $\sim 15 \%$ by purchasing the

443 1728-sample format, and bulk pricing with a 25\% discount of the detection module is available for

$444>10,000$ samples. ** Pricing for the Norgen detection module is based on the 50-sample format

445 running three separate wells (N1, N2 and RNaseP) per sample, pricing can be reduced if purchasing

446 the larger 500-sample format. *** Pricing for SYBR green detection is based on the 200 reaction

447 size LUNA Universal One-Step RT-qPCR Kit (NEB) running three separate wells/sample (two

448 viral genes and one human control gene). Pricing can be reduced up to $30 \%$ with larger kit sizes.

$449 \quad$ N/A: not applicable.

451 Supplementary Figure S1: SYBR green detection of SARS-CoV-2.

452 (A) Standard curves were generated with 8 to 800,000 copies of SARS-CoV-2 RNA (WRCEVA).

453 Mean, $\mathrm{n}=5,2$ independent experiments. (B) Examples of melt curves from a positive high SARS-

454 CoV-2 copy number sample (L024) showing a single specific melt peak, a negative sample (L017)

455 showing non-specific melt peaks, and a positive low SARS-CoV-2 copy number sample (L032)

456 showing both specific and non-specific melt peaks. NTC, no template control (water). 
A
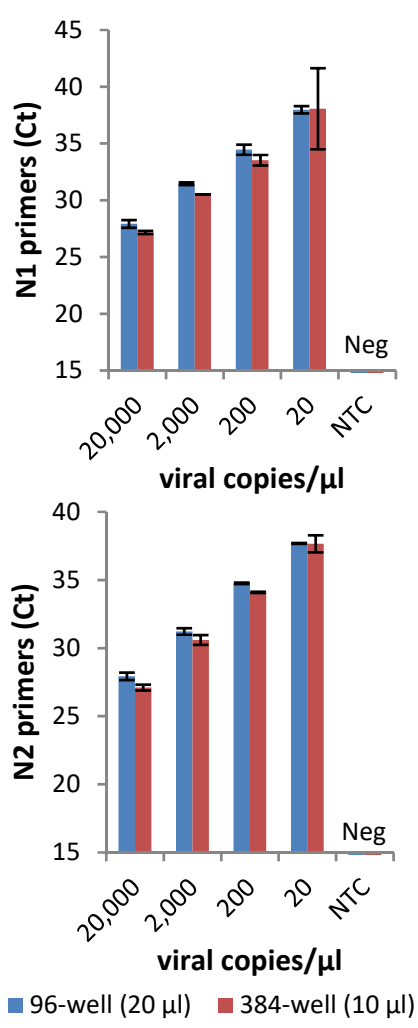

B
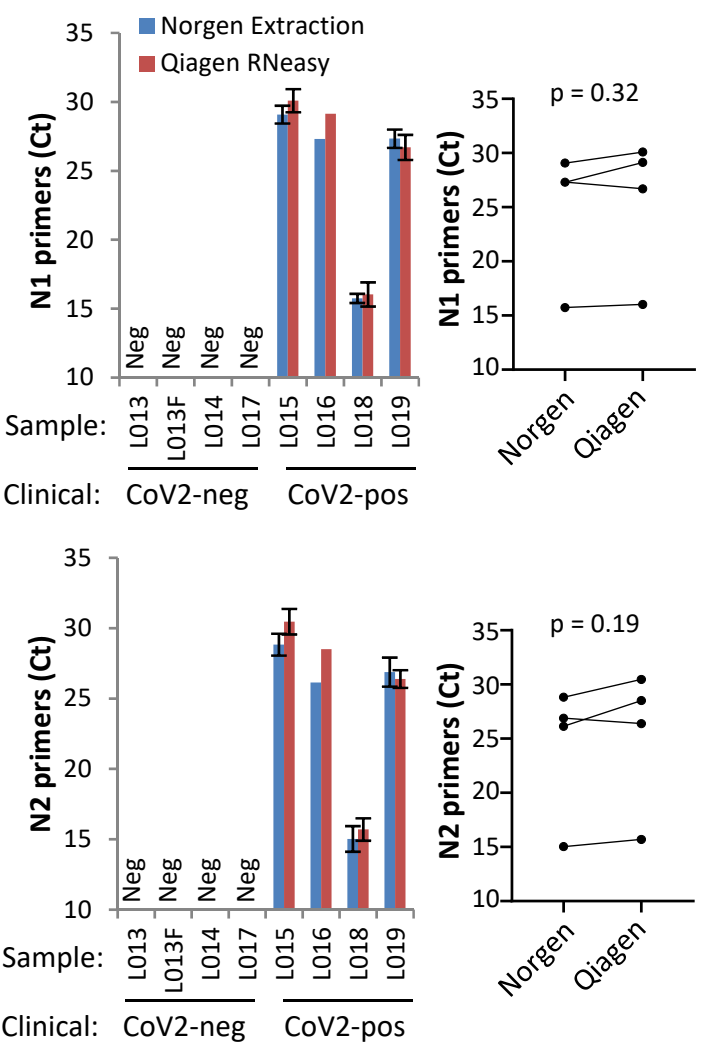

C

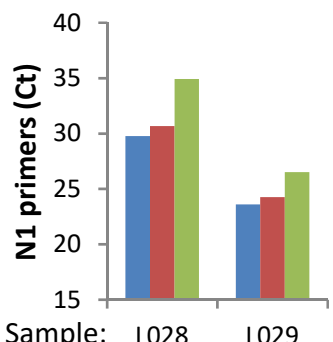

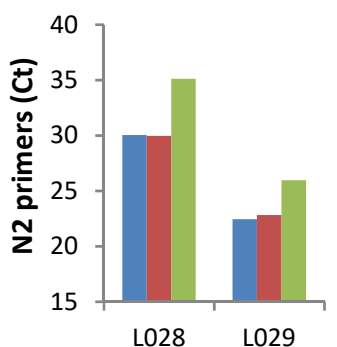

L028 L029

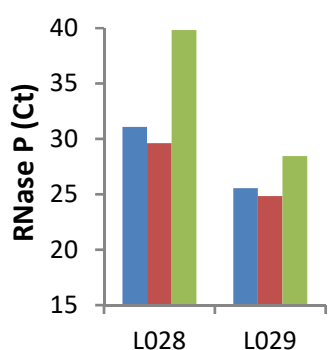

Norgen extraction BGI extraction - Invitrogen extraction

FIG 1: Comparison of plate formats and RNA extraction kits. (A) Serial dilutions of SARS-CoV-2 synthetic RNA standards from Twist Biosci (in copies/ $\mu$ l of the standard added to the RT-qPCR reaction) run in parallel on separate BioRad CFX 96-well (20 $\mu$ reactions) or 384-well (10 $\mu$ l reactions) real-time PCR systems using the Norgen COVID-19 RT-qPCR detection module. Mean $+/$ - range of two independent tests. (B-C) Analysis of four negative and four positive patient samples extracted with either the Qiagen RNeasy or Norgen RNA isolation kits (B) or two positive patient samples extracted with the Norgen, Invitrogen Purelink or BGI RNA isolation kits (C) using the Norgen RT-qPCR detection system and N1, N2 or human control (Rnase P) primers. Samples L015, L018 and L019 are the mean +/- range of technical duplicates run independently on two separate plates, other samples were analyzed once, although L028 and L029 are rerun in Fig. 2a. In (B) a paired t-test was used to compare Norgen vs. Qiagen extractions. 
A

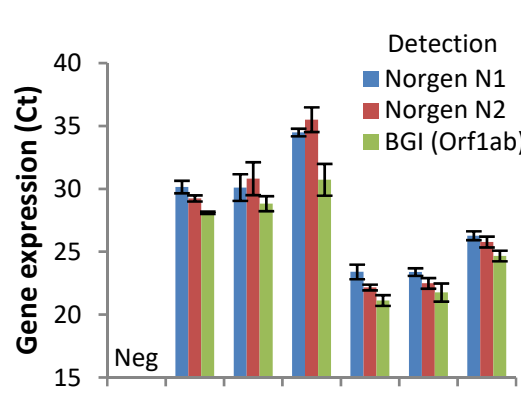

Extraction kit: BGI BGI Nor Pure BGI Nor Pure
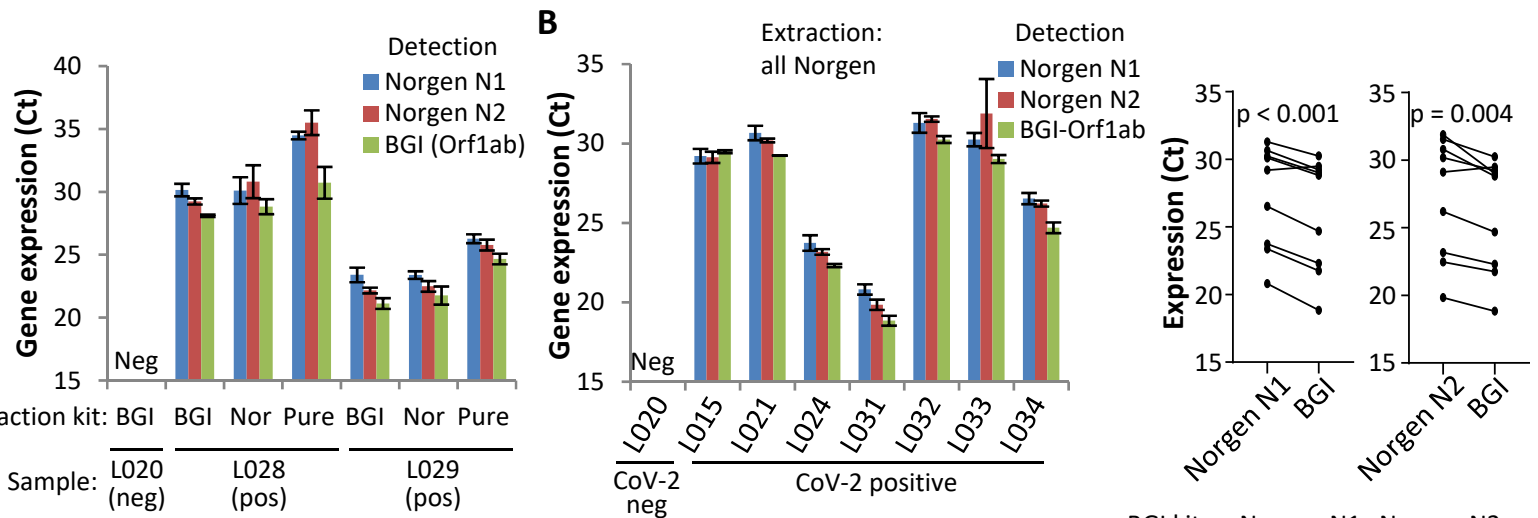

C

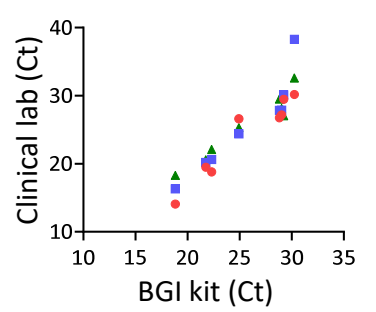

- E gene $\left(R^{2}=0.92, p<0.001\right)$

$\square \operatorname{RdRp}\left(R^{2}=0.85, \mathrm{p}<0.01\right)$

$\triangle N$ gene $\left(R^{2}=0.93, p<0.001\right)$
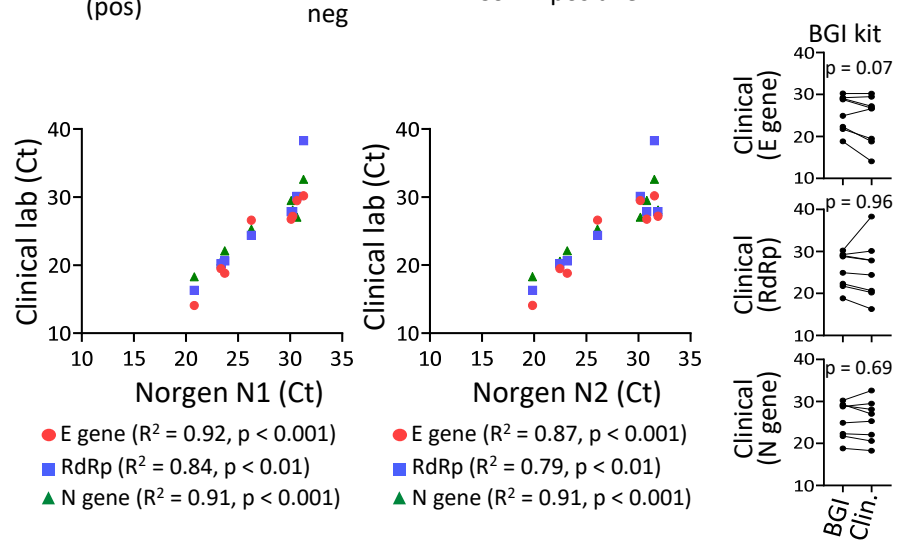

Norgen N1 Norgen N2

D.

\begin{tabular}{|l|c|c|c|c|}
$\begin{array}{r}\text { Detection kit: } \\
\text { Primers: }\end{array}$ & $\begin{array}{c}\text { Norgen } \\
\text { N1 }\end{array}$ & $\begin{array}{c}\text { Norgen } \\
\text { N2 }\end{array}$ & $\begin{array}{c}\text { BGI } \\
\text { Orf1a }\end{array}$ & $\begin{array}{c}\text { Norgen } \\
\text { E_Sarbeco }\end{array}$ \\
\hline 10 copies $/ \mu l$ & $10 / 10$ & $9 / 10$ & $10 / 10$ & $4 / 5$ \\
\hline 5 copies $/ \mu \mathrm{l}$ & $4 / 10$ & $2 / 10$ & $10 / 10$ & $1 / 5$ \\
\hline 2.5 copies $/ \mu \mathrm{l}$ & $3 / 10$ & $3 / 10$ & $9 / 10$ & $2 / 5$ \\
\hline 1 copy $/ \mu \mathrm{l}$ & N/D & N/D & $8 / 10$ & N/D \\
\hline
\end{tabular}

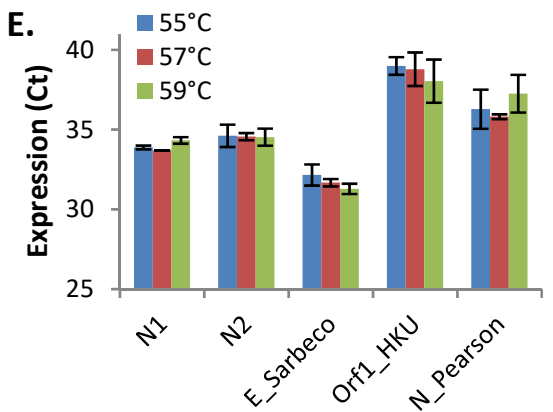

FIG 2: BGI detection kit shows enhanced sensitivity over Norgen kit. (A) Analysis of two positive patient samples extracted with the BGI, Norgen (Nor) or Invitrogen Purelink (Pure) RNA isolation kits using the Norgen (N1 or N2 primers) or BGI RT-qPCR detection systems. Mean +/- std dev of the same sample run on three (BGI \& Norgen extractions) or two (Purelink extraction) separate plates. (B) Analysis of additional patient samples using the Norgen (N1 or N2 primers) or BGI detection systems. Mean +/range of the same samples run independently on two separate plates. Paired t-tests compare Norgen N1 or N2 and BGI Ct values across all samples (including L028 and L029 extracted with the Norgen kit). (C) Comparison of $\mathrm{Ct}$ values from clinical lab analysis (E, RdRp and $\mathrm{N}$ genes) and data obtained with the BGI or Norgen detection systems. Paired t-tests were used to compare results. (D) Detection limit determination using the BGI or Norgen detection systems shown as the number of positives/total number of wells. Concentrations are in copies $/ \mu 1$ in the standard. N/D: not determined. (E) Analysis of 500 viral copies (Twist Biosci) using N1, N2, E Sarbeco, HKU Orf1 and our N gene (N_Pearson) and the Norgen RT-qPCR mix with the indicated annealing/elongation temperatures. Mean $+/$ - range of two independent tests. 
A.

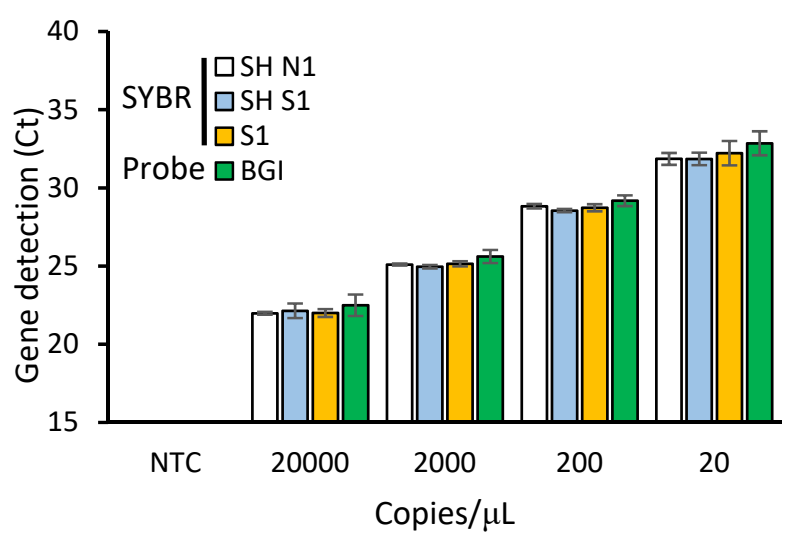

B.

\begin{tabular}{|l|c|c|c|}
\multicolumn{1}{r|}{ Primers: } & S1 & SH N1 & SH S1 \\
\hline 10 copies $/ \mu l$ & $10 / 10$ & $10 / 10$ & $10 / 10$ \\
\hline 5 copies $/ \mu l$ & $9 / 10$ & $9 / 10$ & $9 / 10$ \\
\hline 2.5 copies $/ \mu l$ & $7 / 10$ & $7 / 10$ & $4 / 10$ \\
\hline
\end{tabular}

c.

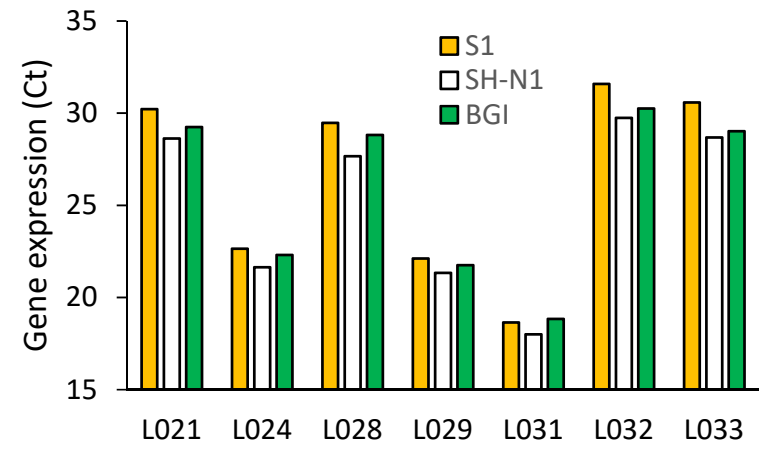

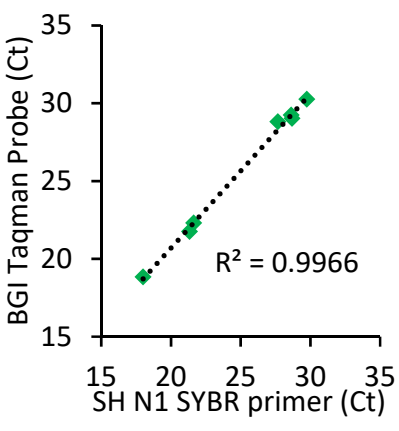

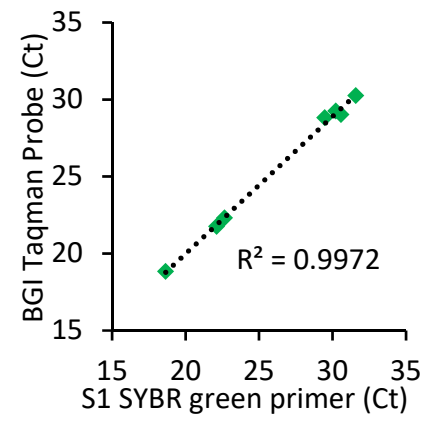

D.

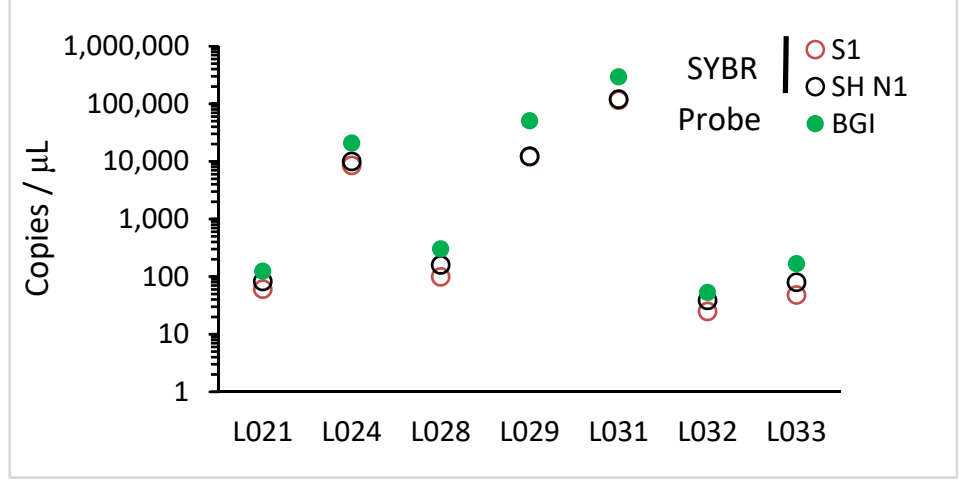

FIG 3. SYBR green detection of extracted RNA. (A) Serial dilutions of SARS-CoV-2 synthetic RNA standards (Twist Biosci) were run in SYBR green and BGI TaqMan assays. Mean $+/-$ STD; $n \geq 3$. (B) Detection limit for each of the SYBR green primer sets shown as the number of positive samples/total number of samples tested. Synthetic RNA (Twist Biosci) was used from stocks with the indicated number of copies per $\mu \mathrm{L}$. (C) Comparison of $\mathrm{Ct}$ values obtained for each patient sample with the SYBR green and BGI TaqMan assays. Linear regression was used to determine the $\mathrm{R}^{2}$. BGI data is from Fig. 2A, B. (D) Comparison of viral copy number per $\mu \mathrm{L}$ for each of the positive patient samples determined with each primer set. Copy number was determined using a standard curve of SARS-CoV-2 RNA (WRCEVA). 
A

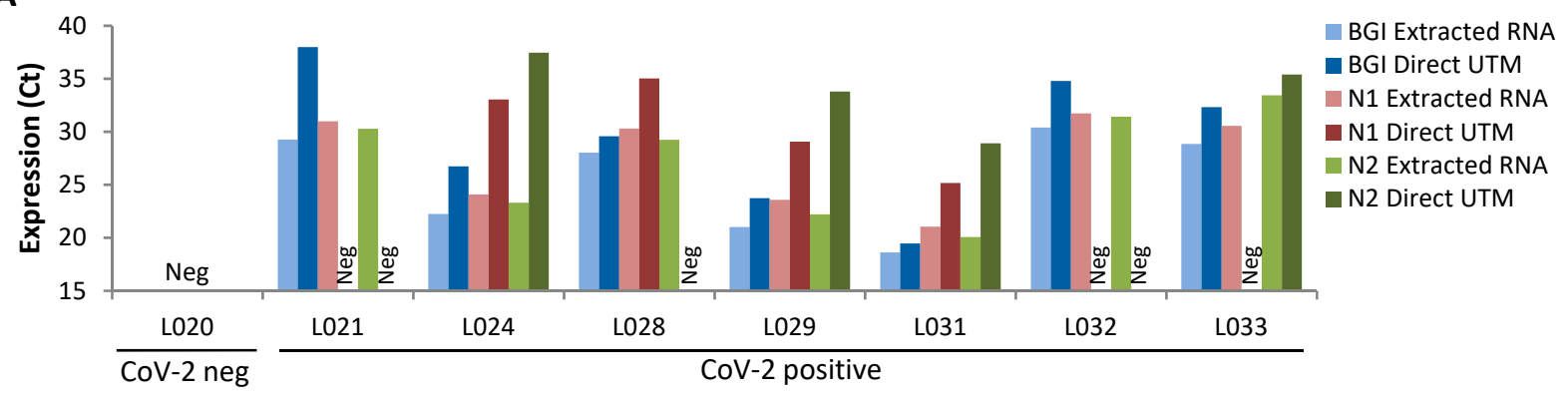

B

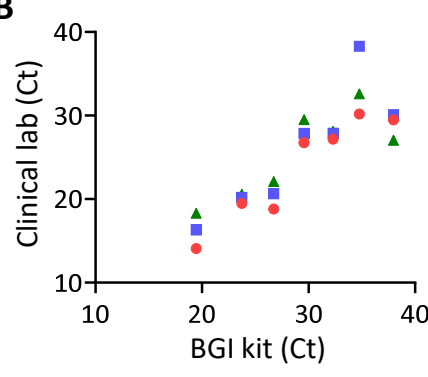

- E gene $\left(\mathrm{R}^{2}=0.90, \mathrm{p}=0.0011\right)$

$\because \operatorname{RdRp}\left(R^{2}=0.76, p=0.0103\right)$

$\triangle N$ gene $\left(R^{2}=0.70, p=0.0193\right)$

D

\begin{tabular}{|l|c|c|c|} 
& $\begin{array}{c}\text { Norgen } \\
\text { System }\end{array}$ & $\begin{array}{c}\text { BGI } \\
\text { System }\end{array}$ & $\begin{array}{c}\text { SYBR } \\
\text { green }\end{array}$ \\
\hline $\begin{array}{l}\text { RNA } \\
\text { extraction } \\
\text { module }\end{array}$ & $\begin{array}{c}\$ 6.55 / \\
\text { sample }\end{array}$ & $\begin{array}{c}\$ 4.68 / \\
\text { sample* }\end{array}$ & N/A \\
\hline $\begin{array}{l}\text { RT-qPCR } \\
\text { detection } \\
\text { module }\end{array}$ & $\begin{array}{c}\$ 2.97 / \\
\text { sample** }\end{array}$ & $\begin{array}{c}\sim 2.58 / \\
\text { sample* }\end{array}$ & $\begin{array}{c}\sim 2.80 / \\
\text { sample*** }\end{array}$ \\
\hline
\end{tabular}

C
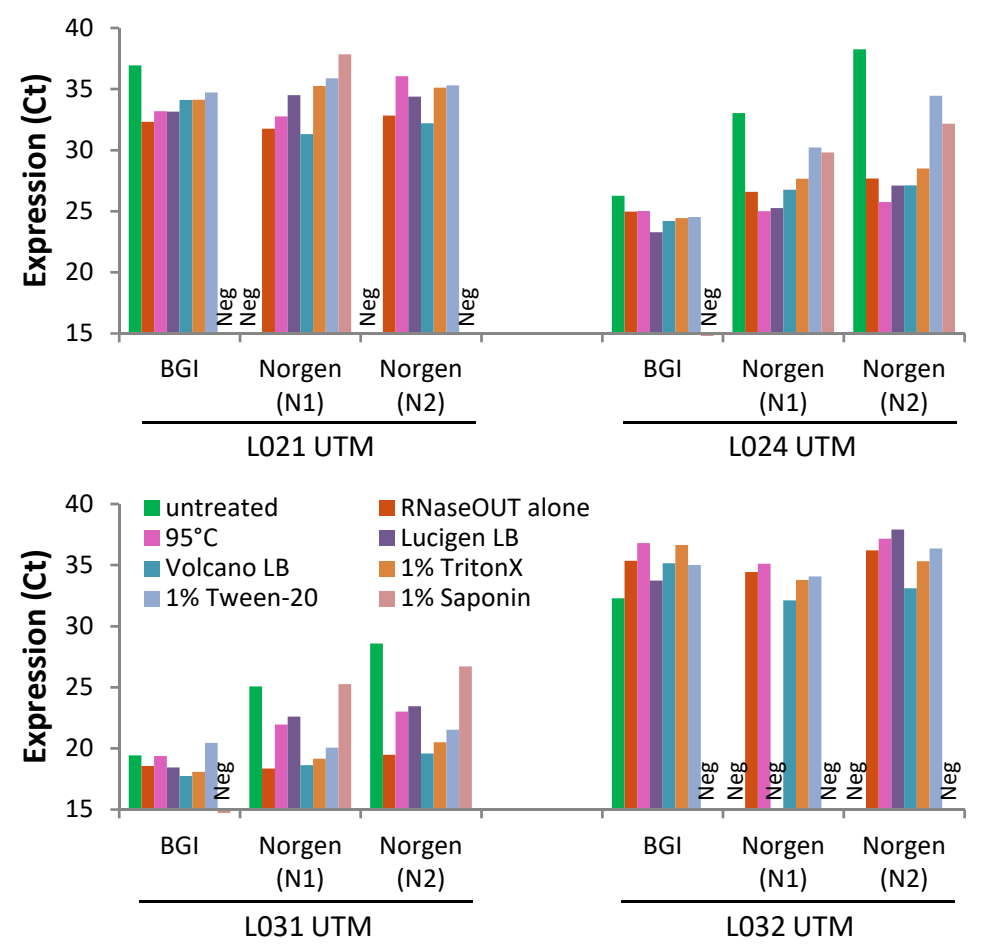

FIG 4: One-step direct detection without RNA extraction. (A) Analysis of extracted RNA or direct UTM from a panel of patients using the BGI or Norgen (N1 and N2 primers) detection systems. (B) Comparison of $\mathrm{Ct}$ values from clinical lab analysis on extracted RNA (E, RdRp and $\mathrm{N}$ genes) to data obtained for direct analysis with the BGI detection system. (C) Patient samples in UTM were left untreated, or treated with the RNase inhibitor RNaseOUT with or without heating at $95^{\circ} \mathrm{C}$ for $15 \mathrm{~min}$, or treated with the indicated lysis buffers/detergents and then directly analyzed using the BGI or Norgen (N1/N2 primers) RT-qPCR detection systems. Note sample L020 (clinical negative) was also tested under these conditions and was confirmed as SARS-CoV-2 negative. (D) Cost analysis comparing Norgen, BGI, and SYBR green systems. Price is in CAD at the time these studies were initiated (late March/early April 2020) for 10 $\mu 1$ RT-qPCR reactions and include relevant processing and shipping fees. * BGI RNA extraction module is based on the 96-sample format, price can be reduced $\sim 15 \%$ by purchasing the 1728 -sample format, and bulk pricing with a $\sim 25 \%$ discount of the detection module is available for $>10,000$ samples. ${ }^{* *}$ Pricing for the Norgen detection module is based on the 50-sample format running three separate wells (N1, N2 and RNaseP) per sample, pricing can be reduced if purchasing the larger 500 -sample format. *** Pricing for SYBR green detection is based on the 200 reaction size LUNA Universal One-Step RT-qPCR Kit (NEB) running three separate wells/sample (two viral genes and one human control gene). Pricing can be reduced up to $30 \%$ with larger kit sizes. N/A: not applicable. 
A.
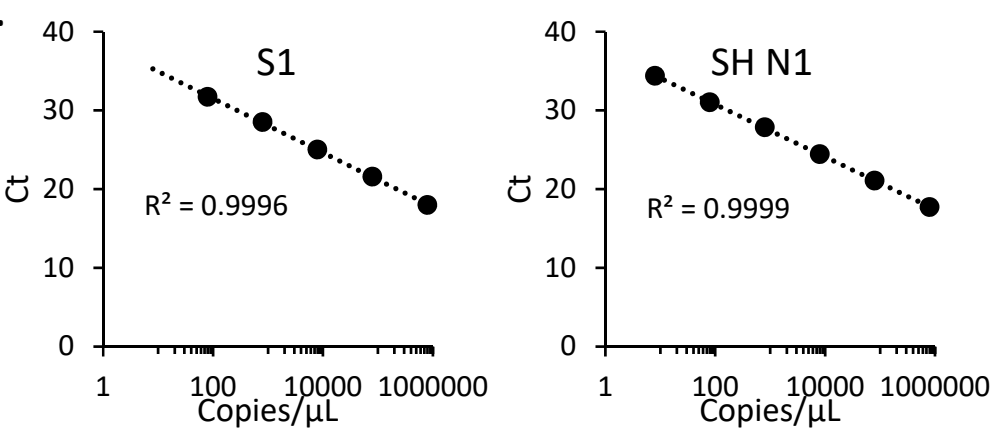

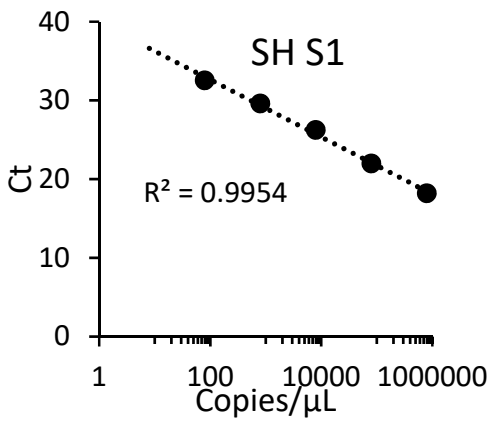

B.
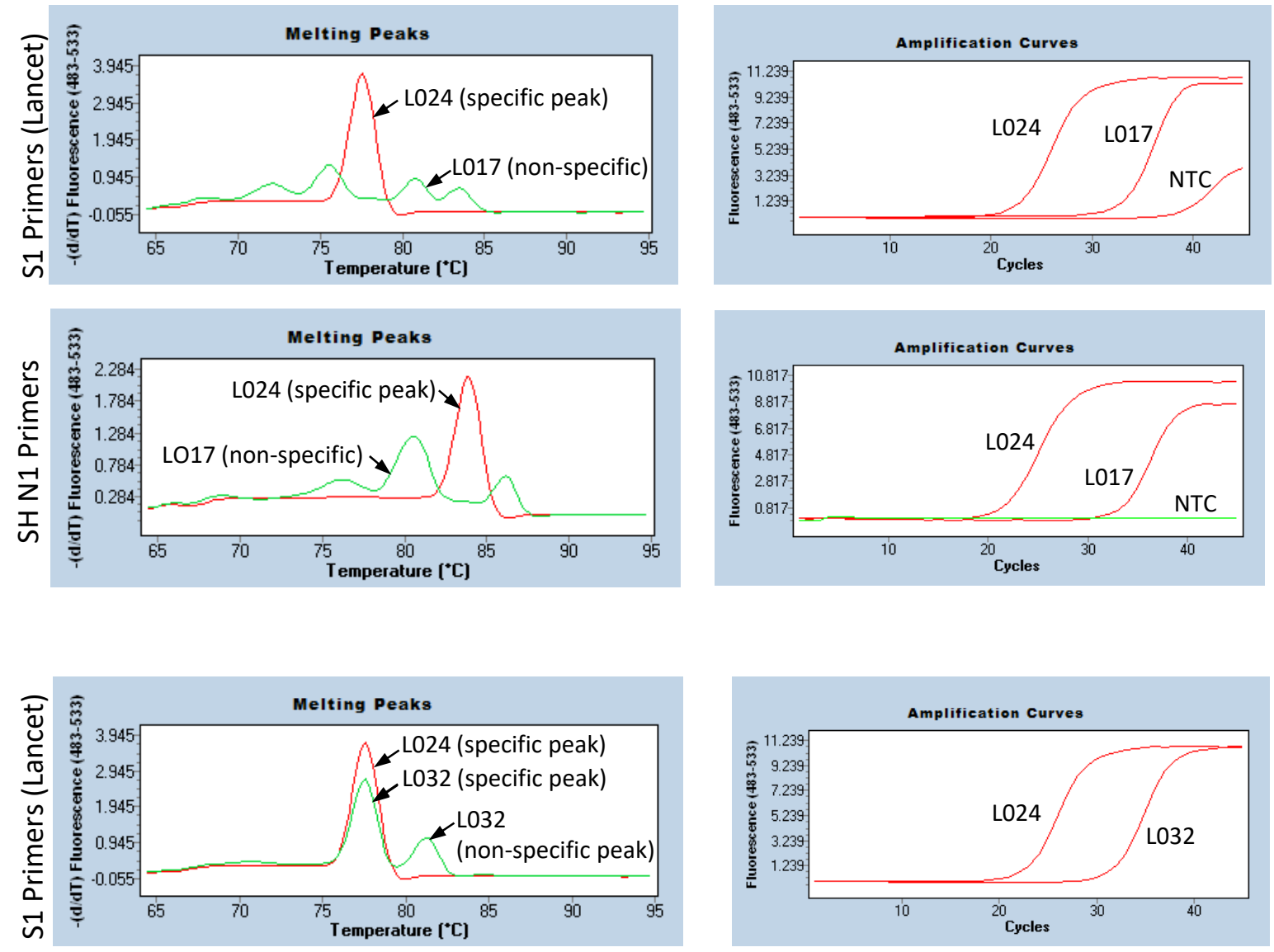

Supplementary Figure S1: SYBR green detection of SARS-CoV-2. (A) Standard curves were generated with 8 to 800,000 copies of SARS-CoV-2 RNA (WRCEVA). Mean, $\mathrm{n}=5,2$ independent experiments. (B) Examples of melt curves from a positive high SARS-CoV-2 copy number sample (L024) showing a single specific melt peak, a negative sample (L017) showing non-specific melt peaks, and a positive low SARS-CoV-2 copy number sample (L032) showing both specific and non-specific melt peaks. NTC, no template control (water). 